\title{
Risk factors and clinical signs of severe Acanthamoeba keratitis
}

This article was published in the following Dove Press journal:

Clinical Ophthalmology

\author{
Machiko Shimmura-Tomita \\ Hiroko Takano \\ Nozomi Kinoshita \\ Fumihiko Toyoda \\ Yoshiaki Tanaka \\ Rina Takagi \\ Mina Kobayashi \\ Akihiro Kakehashi \\ Department of Ophthalmology, \\ Saitama Medical Center, Jichi Medical \\ University, Saitama, Japan
}

Purpose: To determine risk factors and clinical signs for severe Acanthamoeba keratitis (AK) by comparing severe cases with mild cases with good prognosis.

Patients and methods: We reviewed medical records of ten cases of AK (five males and five females) referred to our hospital and classified cases into two groups. One eye that required therapeutic keratoplasty and three eyes with a poor visual acuity $(<0.2)$ on last visit were included in the severe group. Six eyes that had good prognosis with a visual acuity of 1.2 on last visit were classified as mild group. We compared patients' age, the time required for diagnosis, visual acuity on first visit, the history of steroid eye drops use, and other clinical findings.

Results: The average age of the severe group was older than the mild group $(P=0.04)$. The duration between onset and diagnosis of $\mathrm{AK}$ and visual acuity on first visit was not statistically different. A history of steroid eye drop use was found in four eyes of the severe group $(100 \%)$ and four eyes of the mild group (67\%). Keratoprecipitates were found in all severe group eyes and one mild group eye during follow-up $(P=0.01)$. One case in the severe group was diagnosed with diabetes mellitus at initial examination. We detected Staphylococcus epidermis by palpebral conjunctival culture in one case of the severe group.

Conclusion: Aging may be a possible risk factor for severe AK. The presence of keratoprecipitates is a possible sign of severe AK. Attention is also required in patients with comorbidities such as diabetes mellitus and bacterial infection.

Keywords: Acanthamoeba keratitis, keratoprecipitates, steroid eye drops, aging, contact lens

\section{Introduction}

Acanthamoeba keratitis (AK) is a severe and painful corneal infection caused by Acanthamoeba, with a prolonged course featuring remissions and exacerbations. ${ }^{1}$ $\mathrm{AK}$ is an emerging disease with an increasing number of cases presenting each year worldwide, mostly due to the increasing use of contact lens. ${ }^{2}$ Several papers showed that the incidence of AK was recently increasing in UK, India, and New Zealand. ${ }^{3-5}$ Additionally, recent AK cases (2013-2015) were reported to be more severe than previous (2009-2012) cases. ${ }^{6}$ Once established, AK is difficult to treat. Although diagnostic and therapeutic advances have significantly improved overall prognosis, recalcitrant infection and poor outcomes persist in some cases.

Some reports showed that patient-reported duration of symptoms before treatment was reliable in predicting the final visual result and avoiding keratoplasty. ${ }^{7-9}$ Patients with late diagnosis ( $>30$ days) were reported to have a worse prognosis, poor final visual acuity, as well as prolonged disease period. ${ }^{10}$ On the contrary, Alfonso-Muñoz et al reported that the infestation depth (within the deep stroma) at the time of diagnosis appeared to be the main risk factor for requiring penetrating keratoplasty, and Tu et al reported that corneal disease staging at presentation with slit-lamp examination 
was highly predictive. ${ }^{11,12}$ Carnt et al reported that poor AK outcomes were associated with the presence of severe inflammatory complications, aged $>34$ years, corticosteroids used before giving antiamoebic therapy, and symptom duration $>37$ days before antiamoebic therapy. ${ }^{13}$

The prognosis of $\mathrm{AK}$ is important to decide whether aggressive medical or surgical intervention is needed. The purpose of this study was to determine risk factors and clinical signs for severe AK by comparing severe cases with mild cases with good prognosis.

\section{Patients and methods}

This was a retrospective comparative case series in a single referral hospital. We reviewed medical records of consecutive ten cases of AK (five males and five females) referred to Saitama Medical Center, Jichi Medical University, between May 2005 and December 2015. All subjects were treated in accordance with the Declaration of Helsinki. This study was approved by the Institutional Review Board (IRB) of the Jichi Medical University. Written informed consents were obtained from all participants before treatment, as well as additional written informed consents for publication from patients who were described in detail for this study. Patient consent was provided by a parent or legal guardian for any patients under the age of 18 years. According to our IRB approval, patients have the option to opt out of disclosing detailed data including publications. All patients described in the paper have not opted out.

We classified the patients into severe or mild group according to their prognosis. One eye that required therapeutic keratoplasty due to cornea perforation despite intensive conservative therapy and three eyes with slow (over 3 months) response to medical therapy and poor visual acuity $(<0.2)$ on last visit were included in the severe group. Six eyes that showed rapid (within 1 month) response to medical therapy with a best corrected visual acuity (BCVA) of 1.2 on last visit were included in the mild group.

We compared both groups in terms of patient profile, soft contact lens use, systemic disease, the time required for diagnosis, visual acuity on first visit, slit examination on first visit, corneal disease stage on first visit, results of palpebral conjunctiva culture, method of diagnosis, history of steroid eye drops use before diagnosis of AK, existence of keratoprecipitates during follow-up, and cornea scraping frequency. Corneal disease stage was evaluated by slitlamp biomicroscopy and categorized into one to five levels of progressive anatomic involvement and disease severity (epithelitis, epithelitis with radial neuritis, anterior stromal disease, deep stromal keratitis, or ring infiltrate). ${ }^{12}$

\section{Statistical analysis}

Statistical analysis was performed with EZR (Saitama Medical Center, Jichi Medical University, Saitama, Japan), which is a graphical user interface for R (version 2.13.2; The R Foundation for Statistical Computing, Vienna, Austria). ${ }^{14}$ More precisely, it is a modified version of $\mathrm{R}$ commander (version 1.8-4) designed to add statistical functions frequently used in biostatistics. Patient age, gender, contact lens type, systemic disease, slit examination on first visit, the result of palpebral conjunctiva culture, the method of diagnosis, the history of steroid eye drops, and the existence of keratoprecipitates were compared between mild and severe groups using Fisher's exact test. The time required for presentation, the time required for diagnosis, visual acuity, corneal disease stage at first visit, cornea scraping times, and follow-up period were analyzed using the $t$-test. Probability $(P)$ values of $<0.05$ were considered statistically significant.

\section{Results \\ Patient profile}

Patient profiles are shown in Table 1. The mean age of patients was 37 (range 25-51) years old in the severe group and 24 (range $15-35)$ years old in the mild group $(P=0.04)$. One case in the severe group was diagnosed with diabetes mellitus at initial examination (blood sugar level $505 \mathrm{mg} / \mathrm{dL}$, HbA1c value 11.2\%), and another case in the severe group had hypertension. All cases were contact lens users, but they had no history of having entered a pool or a bath while wearing contact lens.

The duration between onset and diagnosis of AK was 240 days in one case of severe group, and in all of the other cases was within 30 days. There was no association between diagnostic delay and severity of AK $(P=0.18)$.

\section{Clinical course}

AK was diagnosed by detection of Acanthamoeba cysts in corneal smears in six eyes or characteristic clinical findings of AK in four eyes (Table 2). BCVA and slit-lamp examination levels at first visit were not significantly different in both groups. Staphylococcus epidermis was detected by palpebral conjunctiva culture in one case of the severe group, which was resistant to topical antibiotics.

A history of steroid eye drops use was found in all eyes (three with $0.1 \%$ betamethasone, one with $0.1 \%$ 
Table I Patients' profile of severe Acanthamoeba keratitis cases group and mild Acanthamoeba keratitis cases group

\begin{tabular}{l|l|l|l|l|l|l}
\hline Case & Age & Gender & Contact lens type & $\begin{array}{l}\text { Systemic } \\
\text { disease }\end{array}$ & $\begin{array}{l}\text { Onset-presentation } \\
\text { (days) }\end{array}$ & $\begin{array}{l}\text { Onset-diagnosis } \\
\text { (days) }\end{array}$ \\
\hline S-I & 36 & Male & 2W DSCL & Diabetes mellitus & 12 & 26 \\
S-2 & $5 I$ & Male & IM DSCL & Hypertension & 15 & 14 \\
S-3 & 25 & Female & IM DSCL & - & 17 \\
S-4 & 35 & Male & DSCL (extended wear) & - & 14 \\
\hline M-I & 28 & Female & 2W DSCL & - & 18 & 240 \\
M-2 & 15 & Female & DSCL & - & 5 & 18 \\
M-3 & 19 & Female & 2W color DSCL & - & 19 & 30 \\
M-4 & 35 & Male & Conventional SCL & - & 12 & 12 \\
M-5 & 17 & Female & DSCL & - & 9 & 9 \\
M-6 & 27 & Male & 2W DSCL & - & 14 & 14 \\
\hline$P$-value & 0.04 & 0.52 & 0.19 & 0.13 & 0.19 & 0.18 \\
\hline
\end{tabular}

Abbreviations: DSCL, disposable soft contact lens; M, month; M-, mild group-; S-, severe group-; W, week.

fluorometholone) of the severe group and four of six eyes (all with $0.1 \%$ fluorometholone) of the mild group (steroid use $P=0.2$, type of steroid use $P=0.07$ ) (Table 3 ). Mean number of cornea scrapings was higher in the severe group $(P=0.07)$.

Keratoprecipitates were found in all eyes of the severe group during follow-up, but only in one eye in the mild group $(P=0.01)$. Keratoprecipitates appeared 3-21 days after stopping steroid eye drops in two eyes of the severe group and one eye of the mild group, but they were observed despite steroid eye drop use in two eyes of the severe group (one eye was on betamethasone, the other eye on fluorometholone). Slit-lamp findings associated with keratoprecipitates were one case of keratoneuritis in the mild group, three cases of ring infiltration (two eyes with steroid eye drops use) in the severe group, and one disciform ulcer in the severe group.

\section{Case presentations}

\section{S-2}

A 51-year-old male was referred to our hospital with keratitis of unknown origin from 11 days prior to visit. He used a monthly type disposable soft contact lens, and his contact lens case was filthy (Figure 1A). He had only been using saline for contact lens care. His BCVA was 0.01 . He had a corneal ring ulcer, Descemet's folds, severe ciliary injection, and severe scleritis (Figure 1B); 0.1\% fluorometholone eye drop and $0.5 \%$ levofloxacin eye drop were prescribed by the former physician. We found Acanthamoeba cysts by corneal smears and $S$. epidermis that was resistant to levofloxacin and other some topical antibiotics by palpebral conjunctiva culture. We discontinued $0.1 \%$ fluorometholone and levofloxacin and started treatment with corneal epithelial debridement, chlorhexidine gluconate eye drops, antifungal eye drops,

Table 2 Patients' data at first visit and diagnostic method of severe and mild Acanthamoeba cases

\begin{tabular}{|c|c|c|c|c|c|}
\hline Case & BCVA & Slit examination & Disease stage & $\begin{array}{l}\text { Conjunctiva } \\
\text { culture }\end{array}$ & Method of diagnosis \\
\hline S-I & 0.7 & Keratoneuritis & 2 & - & Clinical \\
\hline$S-2$ & 0.01 & Ring ulcer & 5 & $\begin{array}{l}\text { Staphylococcus } \\
\text { epidermis }\end{array}$ & Cyst \\
\hline S-3 & 0.6 & Disciform ulcer & 4 & - & Cyst \\
\hline S-4 & 0.8 & $\begin{array}{l}\text { Superficial punctate } \\
\text { keratopathy }\end{array}$ & $\mathrm{I}^{\mathrm{a}}$ & - & Clinical \\
\hline$M-I$ & 0.9 & Pseudo dendritica & 3 & - & Cyst \\
\hline$M-2$ & 0.6 & Keratoneuritis & 2 & - & Clinical \\
\hline M-3 & 0.6 & $\begin{array}{l}\text { Superficial punctate } \\
\text { keratopathy }\end{array}$ & 1 & - & Cyst \\
\hline M-4 & 1.2 & Keratoneuritis & 2 & - & Cyst \\
\hline M-5 & 0.15 & Keratoneuritis & 2 & - & Cyst \\
\hline$M-6$ & 0.9 & Keratoneuritis & 2 & - & Clinical \\
\hline$P$-value & 0.23 & 0.62 & 0.62 & 0.4 & 1 \\
\hline
\end{tabular}

Note: ${ }^{\text {SS}}-4$ had ring ulcer so he was stage 4 when diagnosis.

Abbreviations: BCVA, best corrected visual acuity; M-, mild group-; S-, severe group-. 
Table 3 Steroid eye drop use before diagnosis, the existence of keratoprecipitates during follow-up, cornea scraping times, visual acuity at last visit, and follow-up period

\begin{tabular}{l|l|l|l|l|l}
\hline Case & Steroid drops use & Steroid drops types & Scraping times & Keratoprecipitates & Last visual acuity \\
\hline S-I & + & Betamethasone & 11 & + & $0.8^{\mathrm{a}}$ \\
S-2 & + & Fluorometholone & 26 & + & Hand movement \\
S-3 & + & Betamethasone & 11 & + & 0.2 \\
S-4 & + & Betamethasone & 7 & + & 0.07 \\
\hline M-I & - & & 13 & - & 1.2 \\
M-2 & + & Fluorometholone & 2 & - & 1.2 \\
M-3 & + & Fluorometholone & 5 & - & 1.2 \\
M-4 & + & Fluorometholone & 5 & - & 1.2 \\
M-5 & - & Fluorometholone & 5 & - & 1.2 \\
M-6 & + & 0.07 & + & & 1.2 \\
\hline$P$-value & 0.2 & 0.07 & 0.01 & \\
\hline Note: & & & & 0.12 \\
\hline
\end{tabular}

Note: ${ }^{a}$ After therapeutic keratoplasty.

Abbreviations: M-, mild group-; S-, severe group-

$0.3 \%$ tobramycin eye drop, $0.5 \%$ cefmenoxime eye drop, and systemic antifungal drugs. He presented a disciform infiltration with keratoprecipitates 2 months after referral (Figure 1C). The inflammation eventually tapered off, leaving a corneal scar and mature cataract 12 months after referral (Figure 1D). His visual acuity on last visit was hand movement.

\section{S-4}

A 35-year-old male was referred to our hospital diagnosed with herpetic stromal keratitis that repeated exacerbation and remission from 5 months previously. He was a daily disposable soft contact lens user, but he had worn his contact lens consecutively for 3 days just before the onset of keratitis. His BCVA was 0.8 , and he had superficial punctate keratopathy and corneal stromal infiltration (Figure 1E). Acyclovir ophthalmic ointment and $0.1 \%$ betamethasone eye drop six times a day were prescribed. Corneal smear was negative. Three months after referral, immediately after tapering betamethasone eye drop to three times a day, ring infiltration with keratoprecipitates and severe ciliary injection with severe ocular pain and tearing occurred (Figure 1F). Therefore, we changed the diagnosis to AK based on these clinical findings. After diagnosis, we stopped betamethasone use and started corneal epithelial debridement, chlorhexidine gluconate eye drops, antifungal eye drops, and systemic antifungal drugs. Two years after referral, he had severe corneal scars with no inflammation and his BCVA was 0.07 (Figure 1G).

\section{M-5}

A 17-year-old high school girl was referred to our hospital with superficial corneal stromal opacity and ciliary injection with superficial punctate keratopathy due to contact lens use. She had radial neurokeratitis, pseudodendritic keratitis, superficial stromal infiltration, and iritis on her first visit (Figure 1H). Her BCVA was 0.15. She was diagnosed with AK after amoeba cysts were detected by corneal smear. We treated her with corneal epithelial debridement, chlorhexidine gluconate eye drop, and antifungal eye drops. Three weeks after referral, her BCVA was 1.2 and her cornea was fully recovered (Figure 1I).

\section{Discussion}

In this study, the average age of the severe group was older than the mild group. This result is similar to other reports. ${ }^{6,13}$ This may reflect the fact that aging changes the function of the systemic immune system ${ }^{15}$ and may lead to severe keratitis in the elderly. One patient had diabetes in the severe group, which is well-known as a risk factor for infectious keratitis. ${ }^{16}$ However, systemic diseases were not associated with severity of AK in this study.

We could not find an association between contact lens type and severity of AK in this case series because of too much variation in the type of contact lens used. However, it is worth noting that all our cases were contact lens wearers. The development of AK in contact lens users is strongly related to poor lens hygiene and contaminated water., ${ }^{210,13,17-21}$ In this study, lens care was normal in most patients, but one case in the severe group (S-2) had only been using saline for contact lens care and his contact lens case was filthy. Poor contact lens hygiene may be a risk factor for severe AK. The overnight wear of lenses remains the dominant risk factor for the development of $\mathrm{AK}^{, 2,23}$ but only one case in our severe group (S-4) used extended wear lens. The use of contact lens while swimming or bathing is reported to be a risk factor for $\mathrm{AK},{ }^{13}$ but there were no cases of using contact lens as such in our study. 

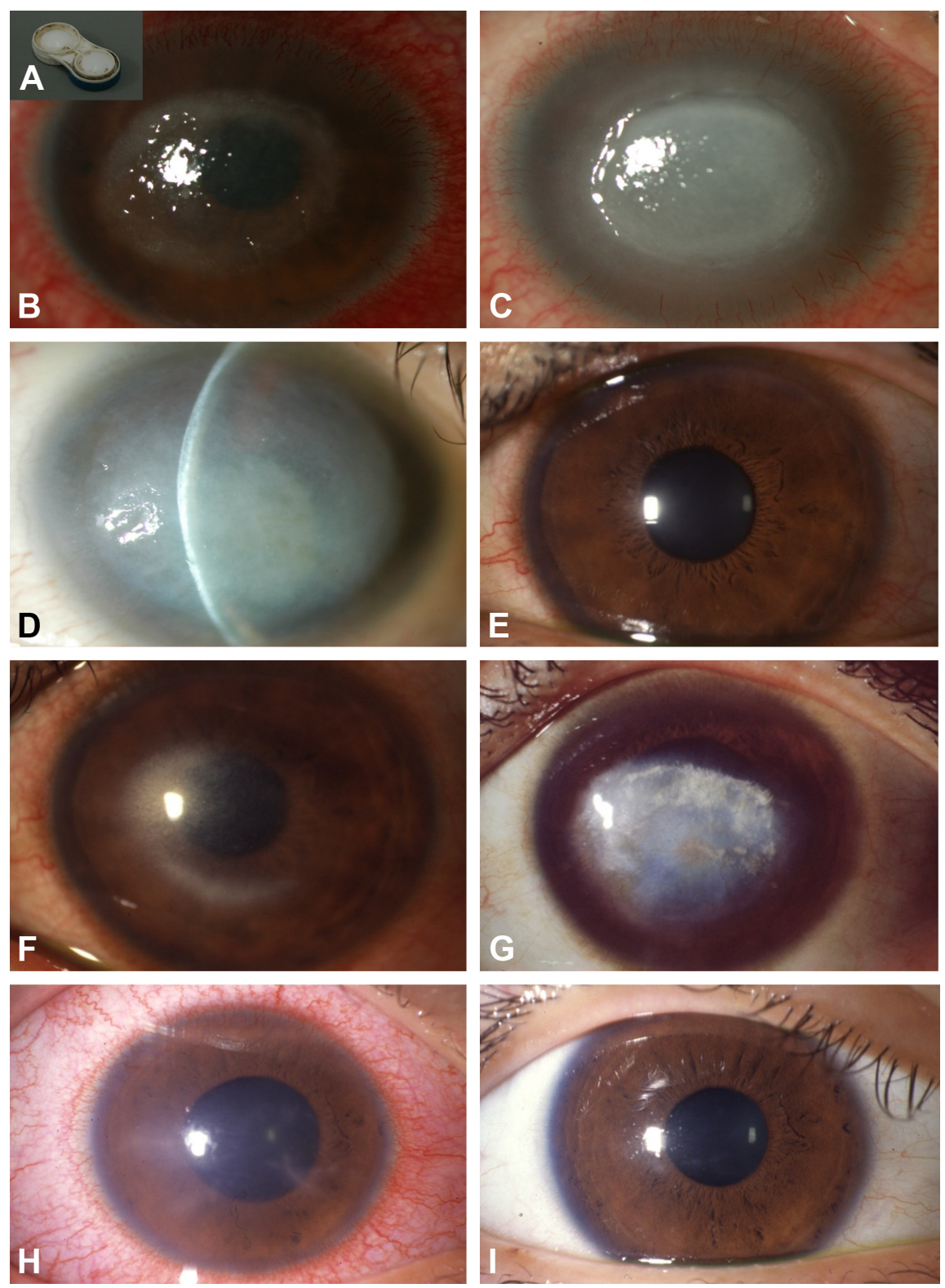

Figure I Slit-lamp photographs.

Notes: (A-D) Case S-2. (A) Filthy soft contact lens case, (B) ring ulcer at first visit, (C) disciform infiltrations with keratoprecipitates at 2 months after referral, and (D) corneal scar and mature cataract at 12 months after referral. (E-G) Case S-4. (E) Superficial punctate keratopathy at first visit, (F) ring infiltration with keratoprecipitates at 3 months after referral, and (G) corneal scar at 2 years after referral. ( $\mathbf{H}$ and $\mathbf{I})$ Case $\mathbf{M}-5$. (H) Keratoneuritis and ciliary injection at first visit, and $(\mathbf{I})$ clear cornea at 3 weeks after referral.

Some papers report that an early diagnosis and early start of antiamoebic treatment result in a better final visual acuity, ${ }^{4,6-10,13}$ however, nine out of ten of our patients were diagnosed within 1 month after the onset of AK. There was no association between diagnostic delay and severity of AK in our study. This suggests that there may be other important factors for AK prognosis than early diagnosis.

Visual acuity at presentation was not associated with outcome. Furthermore, corneal disease stage and slit-lamp examination at presentation were not associated with prognosis in our case series, which differed from a previous study, suggesting that corneal disease staging at presentation was highly predictive of outcomes. ${ }^{11,12}$ However, eight out of ten patients in our series used steroid eye drops before diagnosis, and therefore, visual acuity and slit-lamp examination may have been masked. Furthermore, S- 4 only had superficial punctate keratopathy at first visit and was diagnosed as stage 1; however, he had a ring ulcer when AK was diagnosed, after which he was diagnosed as stage 5. Therefore, staging at diagnosis may have a major impact on prognosis. 
Severe cases tended to use betamethasone eye drops, and mild cases tended to use fluorometholone eye drops before diagnosis, but there was no significant difference. Betamethasone use may have triggered a bad prognosis. On the contrary, strong inflammation in the severe group may have prompted the physician to prescribe betamethasone eye drops. Because there was no delay in diagnosis of the severe group, we believe that strong inflammation was the reason for betamethasone use.

Keratoprecipitates were found in all severe group eyes and one eye of the mild group ( $P=0.01)$. Keratoprecipitates are signs of deep infiltration of Acanthamoeba associated with inflammation in the anterior chamber. Keratoprecipitates may be associated with the use of betamethasone in the severe group because of strong inflammation. The reasons for deep amoeba infiltration may be due to: 1) immune suppression by using steroid eye drops; 2) patients with comorbidities such as age, diabetes, and mixed infection. We examined the relationship of keratoprecipitates presentation and steroid eye drop use. Two eyes in the severe group and one eye in the mild group showed keratoprecipitates 3-21 days after stopping steroid eye drops. This is most likely due to the fact that steroid eye drops masked keratoprecipitates appearance or suppressed immune reaction. Interestingly, another two eyes in severe group had keratoprecipitates regardless of using steroid eye drops (betamethasone: one eye, fluorometholone: one eye). The presence of keratoprecipitates while using steroid eye drops may be a sign of very severe Acanthamoeba infection.

The resistant bacteria in case S-2 may have been a nutrient source for Acanthamoeba. Acanthamoeba may proliferate by consuming bacteria. In this case, coinfection of bacteria may have been one reason of the severity of AK. This particular case in the severe group was interesting because the presence of bacteria in $\mathrm{AK}$ was reported to be a requirement for the development of $\mathrm{AK}^{2}{ }^{24}$

\section{Limitation}

The limitation of this study is the small number of cases. This may be due to the fact that the number of AK patients has recently been declining in Japan due to increasing public awareness on proper contact lens use publicized by ophthalmic societies. ${ }^{25}$ If more cases were examined, a more detailed investigation on prognostic factors may be possible.

\section{Conclusion}

Aging may be a possible risk factor for severe AK, and the presence of keratoprecipitates especially while using corticosteroids eye drops is a possible sign of severe AK. Attention is also required in patients with comorbidities such as diabetes mellitus and bacterial infection.

\section{Acknowledgment}

The authors would like to thank Dr Shigeto Shimmura of the Department of Ophthalmology of Keio University School of Medicine for English editing support.

\section{Author contributions}

All authors contributed to data analysis, drafting and revising the article, gave final approval of the version to be published, and agree to be accountable for all aspects of the work.

\section{Disclosure}

The authors report no conflicts of interest in this work.

\section{References}

1. Maycock NJ, Jayaswal R. Update on Acanthamoeba keratitis: diagnosis, treatment, and outcomes. Cornea. 2016;35(5):713-720.

2. Juárez MM, Tártara LI, Cid AG, et al. Acanthamoeba in the eye, can the parasite hide even more? Latest developments on the disease. Cont Lens Anterior Eye. 2018;41(3):245-251.

3. Carnt N, Hoffman JJ, Verma S. Acanthamoeba keratitis: confirmation of the UK outbreak and a prospective case-control study identifying contributing risk factors. Br J Ophthalmol. Epub 2018 Sep 19.

4. Lalitha P, Lin CC, Srinivasan M, et al. Acanthamoeba keratitis in South India: a longitudinal analysis of epidemics. Ophthalmic Epidemiol. 2012; 19(2):111-115.

5. Mckelvie J, Alshiakhi M, Ziaei M, Patel DV, Mcghee CN. The rising tide of Acanthamoeba keratitis in Auckland, New Zealand: a 7-year review of presentation, diagnosis and outcomes (2009-2016). Clin Exp Ophthalmol. 2018;46(6):600-607.

6. Roozbahani M, Hammersmith KM, Rapuano CJ, Nagra PK, Zhang QE, Siu SY. Acanthamoeba keratitis: are recent cases more severe? Cornea. 2018;37(11):1381-1387.

7. Claerhout I, Goegebuer A, van den Broecke C, Kestelyn P. Delay in diagnosis and outcome of Acanthamoeba keratitis. Graefes Arch Clin Exp Ophthalmol. 2004;242(8):648-653.

8. Dart JK, Saw VP, Kilvington S. Acanthamoeba keratitis: diagnosis and treatment update 2009. Am J Ophthalmol. 2009;148(4):e482: 487-499.

9. Illingworth CD, Cook SD, Karabatsas CH, Easty DL. Acanthamoeba keratitis: risk factors and outcome. Br J Ophthalmol. 1995;79(12): 1078-1082.

10. Lee MH, Abell RG, Mitra B, Ferdinands M, Vajpayee RB. Risk factors, demographics and clinical profile of Acanthamoeba keratitis in Melbourne: an 18-year retrospective study. Br J Ophthalmol. 2018;102(5): 687-691.

11. Alfonso-Muñoz EA, Roig-Revert MJ, Fernández-López E, HernándezDíaz M, Araujo-Miranda R, Peris-Martínez C. A report of 10 patients with Acanthamoeba keratitis. Arch Soc Esp Oftalmol. 2018;93(10):497-502.

12. Tu EY, Joslin CE, Sugar J, Shoff ME, Booton GC. Prognostic factors affecting visual outcome in Acanthamoeba keratitis. Ophthalmology. 2008;115(11):1998-2003.

13. Carnt N, Robaei D, Minassian DC, Dart JKG. Acanthamoeba keratitis in 194 patients: risk factors for bad outcomes and severe inflammatory complications. Br J Ophthalmol. 2018;102(10):1431-1435.

14. Kanda Y. Investigation of the freely available easy-to-use software 'EZR' for medical statistics. Bone Marrow Transplant. 2013;48(3):452-458. 
15. Denkinger MD, Leins H, Schirmbeck R, Florian MC, Geiger H. HSC aging and senescent immune remodeling. Trends Immunol. 2015; 36(12):815-824.

16. Badawi AE, Moemen D, El-Tantawy NL. Epidemiological, clinical and laboratory findings of infectious keratitis at Mansoura Ophthalmic Center, Egypt. Int J Ophthalmol. 2017;10(1):61-67.

17. Jeong HJ, Lee SJ, Kim JH, et al. Acanthamoeba: keratopathogenicity of isolates from domestic tap water in Korea. Exp Parasitol. 2007;117(4): 357-367.

18. Joslin CE, Tu EY, Mcmahon TT, Passaro DJ, Stayner LT, Sugar J. Epidemiological characteristics of a Chicago-area Acanthamoeba keratitis outbreak. Am J Ophthalmol. 2006;142(2):212-217.

19. Joslin CE, Tu EY, Shoff ME, et al. The association of contact lens solution use and Acanthamoeba keratitis. Am J Ophthalmol. 2007;144(2): $169-180$

20. Kilvington S, Gray T, Dart J, et al. Acanthamoeba keratitis: the role of domestic tap water contamination in the United Kingdom. Invest Ophthalmol Vis Sci. 2004;45(1):165-169.
21. Radford CF, Minassian DC, Dart JK. Acanthamoeba keratitis in England and Wales: incidence, outcome, and risk factors. Br J Ophthalmol. 2002; 86(5):536-542.

22. Keay L, Edwards K, Dart J, Stapleton F. Grading contact lens-related microbial keratitis: relevance to disease burden. Optom Vis Sci. 2008; 85(7):531-537.

23. Stapleton F, Keay L, Edwards K, et al. The incidence of contact lensrelated microbial keratitis in Australia. Ophthalmology. 2008;115(10): 1655-1662.

24. Nakagawa H, Hattori T, Koike $\mathrm{N}$, et al. Investigation of the role of bacteria in the development of acanthamoeba Keratitis. Cornea. 2015; 34(10):1308-1315.

25. Toriyama K, Suzuki T, Ohashi Y. Survey of the number of Acanthamoeba keratitis cases in Japan. Nippon Ganka Gakkai Zasshi. 2014; 118(1):28-32.
Clinical Ophthalmology

\section{Publish your work in this journal}

Clinical Ophthalmology is an international, peer-reviewed journal covering all subspecialties within ophthalmology. Key topics include: Optometry; Visual science; Pharmacology and drug therapy in eye diseases; Basic Sciences; Primary and Secondary eye care; Patient Safety and Quality of Care Improvements. This journal is indexed on

Submit your manuscript here: http://www.dovepress.com/clinical-ophthalmology-journal

\section{Dovepress}

PubMed Central and CAS, and is the official journal of The Society of Clinical Ophthalmology (SCO). The manuscript management system is completely online and includes a very quick and fair peer-review system, which is all easy to use. Visit http://www.dovepress.com/ testimonials.php to read real quotes from published authors. 